\title{
The Future Internet Enablement of the Environment Information Space
}

\author{
Thomas Usländer ${ }^{1}$, Arne J. Berre ${ }^{2}$, Carlos Granell ${ }^{3}$, \\ Denis Havlik ${ }^{4}$, José Lorenzo ${ }^{5}$, Zoheir Sabeur ${ }^{6}$, and Stefano Modafferi ${ }^{6}$ \\ ${ }^{1}$ Fraunhofer IOSB, Germany \\ thomas.uslaender@iosb. fraunhofer.de \\ ${ }^{2}$ SINTEF, Norway \\ arne.j.berreasintef . no \\ ${ }^{3}$ European Commission, Joint Research Centre, Italy \\ carlos.granell@jrc.ec.europa.eu \\ ${ }^{4}$ Austrian Institute of Technology (AIT) \\ Denis.Havlik@ait.ac.at \\ ${ }^{5}$ Atos, Spain \\ jose.lorenzo@atosresearch.eu \\ ${ }^{6}$ University of Southampton IT Innovation Centre, \\ Faculty of Physical Sciences and Engineering, United Kingdom \\ \{zas, sm\}@it-innovation. soton.ac.uk
}

\begin{abstract}
This paper motivates the enablement of the Future Internet to become a highly functional service platform supporting the design and the operation of software applications in the Environmental Information Space. It reports on the experience made by the European research project ENVIROFI as one of the usage area projects within the Future Internet Public-Private Partnership programme. It describes the software components (environmental and specific enablers) which are required to connect with the domain-independent capabilities (generic enablers) of the Future Internet core platform for geospatially and environmentally-driven applications.
\end{abstract}

Keywords: Future Internet, ENVIROFI, generic enablement, specific enablement, environment information space, Open Geospatial Consortium, OGC.

\section{Motivation}

Starting from proprietary software frameworks and the societal need for interoperable environmental services, the object- and service-orientated architecture design approaches of the past have paved the way to open, standardized and distributed design of the Environmental Information Space (EIS) [1]. The emerging Future Internet (FI) platform aims to establish an even more generic set of software building blocks which simplify the building of cross-domain applications.

With the mainstream trends towards virtually centralized computing resources (cloud computing) combined with the predominant role and extension of the Internet 
technology towards an Internet of Things (IoT), Content, Services and People, EIS stakeholders are facing a disruptive technology shift which will affect the established technologies as well as the business models of EIS. The environmental data generated by smart low-cost sensors, also known as "Things" in the IoT context, is getting increasingly ubiquitous instead of being offered as distinguished "products" by service providers in the environmental domain sectors. Smart processing capabilities, e.g. the provision of aggregated environmental data as thematic layers in Geographic Information Systems (GIS), can be used on demand. The intention to combine huge amounts of environmental sensor-based observations with unstructured or semistructured information of Web documents (e.g. environmental reports, images, videos) as well as with the human observations posted in social media transforms the design requirements of future EIS and the analysis of the existing sources of environmental information into "Big Data" management requirements.

In order to profit from these development, EIS stakeholders (e.g. designers, implementers) have to face the challenging task of integrating the legacy and future EIS applications into the emerging Future Internet framework. They need to understand the current and upcoming user requirements [2], often expressed by thematic experts of a particular domain, and to map these requirements onto highly generic FI software building blocks. In order to assure their re-use and sustainable implementations in context of the FI Enablement, such building blocks shall be specified and developed in an agile way, feature simple and well-defined services interfaces, and follow existing standards whenever possible. This sets the requirements for building the new generation of EIS to higher grounds once again while agile development methodologies and new domain-independent FI software building blocks have to be embraced.

Past experiences with the EIS design have stressed the fact that the majority of environmental information objects have geospatial references. This is reflected in the European regulations on information sharing and spatial data infrastructures such as INSPIRE, as well as in the widespread use of standards promoted by the Open Geospatial Consortium (OGC) within the environmental usage area. Hence, the building blocks of such an open and FI-enabled EIS have to support the geospatial processing as well as the existing open standards for geospatial data and service interfaces.

The rest of the paper is organized as follows. Section 2 of this paper introduces the FI-PPP programme and its environmental usage area project ENVIROFI. Three environmental pilot applications pursued by ENVIROFI are introduced in Section 3, followed by the presentation of the ENVIROFI Conceptual Architecture and the discussion of the generic and specific enablers in sections 4 and 5 respectively. The encountered technical challenges and the lessons learnt during the project are discussed in section 6. Finally, section 7 summarizes the vision of the future development of an FI-enabled EIS and environmentally enabled FI.

\section{The Future Internet Public-Private Partnership}

The European Commission (EC) launched the Future Internet Public-Private Partnership programme (FI-PPP) with the idea of enabling a broad range of Internet applications, including those of the environmental knowledge domain. The FI-PPP programme shall advance a shared vision for common pan-European technology 
platforms and their implementation, as well as the integration and harmonization of the relevant policy, legal, and regulatory frameworks. As set in the Digital Agenda for Europe $^{1}$, these frameworks are prerequisites for realizing a common European digital market economy with inclusive access to knowledge and awareness to societal issues. Phase 1 of the FI-PPP started a set of interrelated projects, addressing different use cases in vertical domain sectors. They cooperated with the ICT Core Platform which is developed in the FI-WARE project (see http://www.fi-ware.eu/).

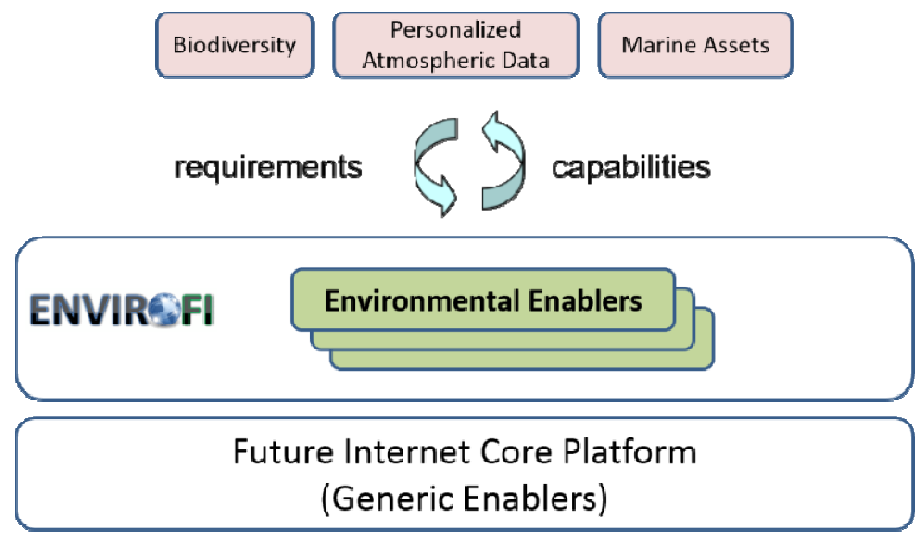

Fig. 1. ENVIROFI Approach to Design an Environmental Observation Web

As illustrated in Fig. 1 the FI-WARE platform is an ICT platform based on generic functional building blocks: the so called Generic Enablers (GE). These are used by the usage area projects in order to validate and demonstrate the new generation FI enabled environmental applications. Furthermore, the project provides development benchmarks for innovation schemes and guiding new market sector Enterprise buy-in FI technologies. ENVIROFI represents the environmental usage area within phase 1 of FI-PPP (see http://www.envirofi.eu/). It explores the so-called environmental enablers, i.e. reusable building blocks for collecting and processing environmental data, and provides environmental sector requirements to FI-WARE. Thus, ENVIROFI lays the foundation for an FI-enabled Environmental Observation Web, which will help Europe to tackle the grand challenges of climate change, socio-economic pressures on the environment and sustainable development.

ENVIROFI's vision is to establish an Environmental Observation Web in which all environmental data from sensors, citizens and models become available through the Internet in a standardized and usable format. ENVIROFI specifically works on three environmental application areas: biodiversity, personalized atmospheric data and marine assets.

${ }^{1}$ http: //ec.europa.eu/digital-agenda/ 


\section{Pilot Applications}

The identification of the specific building blocks and enablers for the environment has been guided by an agile service-oriented analysis and design methodology [1, 3] of the EIS requirements inherited from existing European initiatives such as Copernicus ${ }^{2}$ and INSPIRE ${ }^{3}$, and the more specific requirements of the following pilot applications.
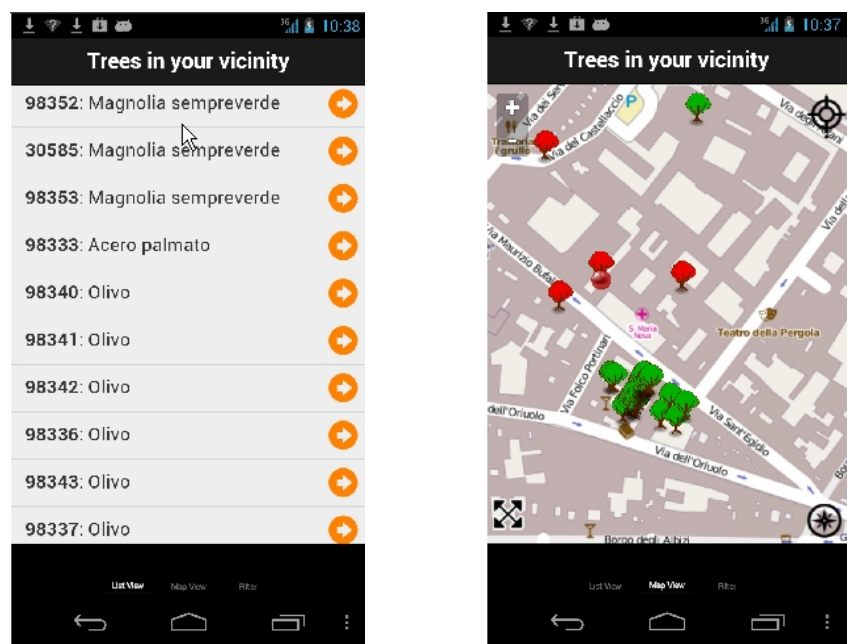

Fig. 2. Screenshots of the BIO app: The left hand side screenshot shows a list of trees around a given user's location. The right hand side screenshot shows such information on a map of the City of Florence (Italy).

1. Bringing Biodiversity into the Future Internet: This pilot focuses on the usage of FI technology for the survey, analysis, quality assurance, persistence and dissemination of biodiversity data. The Biodiversity Survey Application (BIO App) aims to support the monitoring of terrestrial biodiversity. This enables users from a wide variety of backgrounds to: (i) Access data on interesting biodiversity occurrences which are recorded at their geographic location; (ii) Provide data on biodiversity occurrences which they observe; and (iii) Provide feedback on the correctness of existing and new recorded biodiversity occurrence in the system. In order to allow data provisioning by users, they may either add information to existing entries on individual plants or animals, or provide information on specimen which are not contained within the system. Although the BIO App operates in any geographical region, sample data sets in the cities of Vienna (Austria) and Florence (Italy) (see Fig. 2) allow users to test with large data sets, based on open data tree layers which are made available.

\footnotetext{
${ }^{2}$ Previously known as GMES (Global Monitoring for Environment and Security), see http: / / copernicus.eu/

${ }^{3}$ Infrastructure for Spatial Information in the European Community, see http://inspire.jrc.ec.europa.eu/
} 


\section{Personal Information System for Air Pollutants, Allergens and Meteorological} conditions: This pilot focuses on the interaction with the environment by creating a series of integrated tools that comprise the backbone of the Personal Information System for Air Pollutants, Allergens and Meteorological conditions which potentially affect human health and well-being. The Personal Environmental Information System (PEIS) is a fully customizable, interactive mobile application enabling users to monitor information on weather conditions and atmospheric exposures and related hazards from their own perspective. PEIS was tested for the cities of Oslo (Norway) and Vienna (Austria) (see Fig. 3).
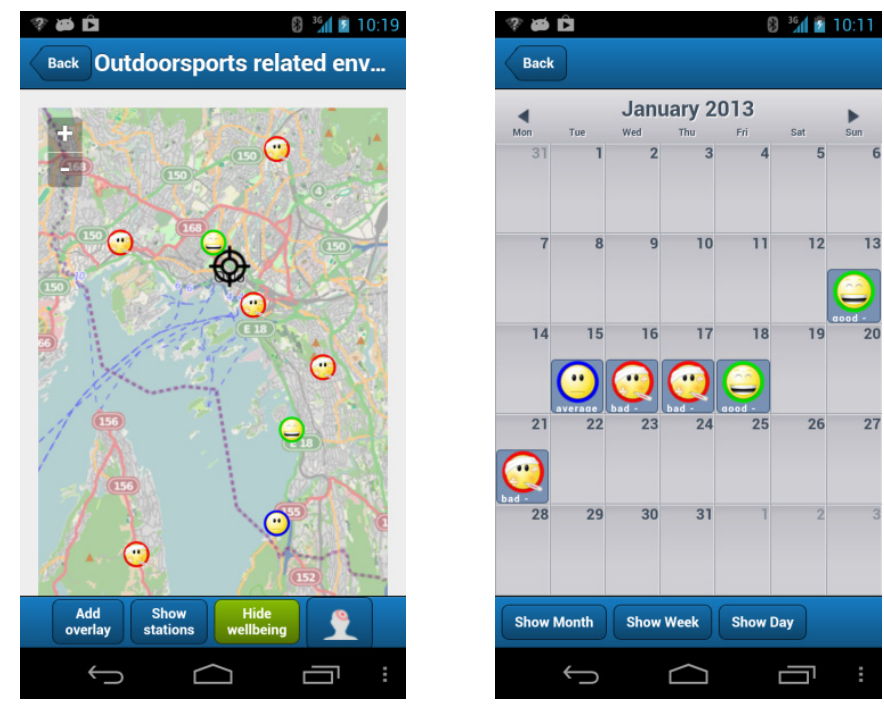

Fig. 3. Screenshots of the PEIS app: The left hand side screenshot shows a map of health and environmental conditions at outdoor sport facilities in the city of Oslo (Norway). The right hand side screenshot shows a personalized health calendar.

3. Future Internet Collaborative Usage of Marine Environmental Assets: This pilot focuses on the collaborative use of FI technology for Marine Environmental Assets. The Marine Asset decision Support Tool (MAST) is a Web portal that mashes up a number of freely available information and data feeds to monitor operational weather conditions in the Galway Bay, Ireland, promoting situational awareness among offshore marine operators. Through the intelligent fusion of realtime observation data with model predictions, the portal can be used to identify monitor sea-state conditions, water quality and the encountered risks for the marine industry to operate at sea. These include the aquaculture, fishing and leisure industries (see Fig. 4). 


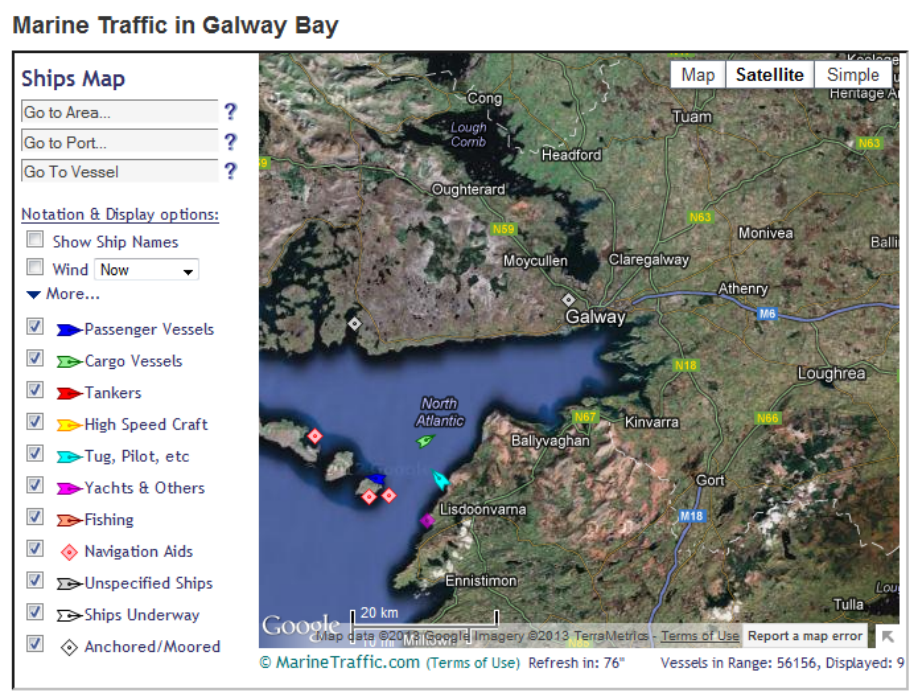

Fig. 4. Screenshots of the MAST portal: it shows real time conditions collected from various observation platforms around Galway Bay

\section{Conceptual Architecture}

The FI-WARE technical chapters are: Cloud Hosting, Data/Context Management, Internet of Things (IoT) Services Enablement, Applications/Services Ecosystem and Delivery Framework, Security and Interface to Networks and Devices (I2ND). They are highly independent from each other, and may heavily differ in terms of the adopted standards, technologies, data models, data representations and interaction patterns. They are application and use case agnostic to the level where none of the semantics that is implicitly available in the Use Case projects has been a-priori known to the FI-WARE GEs. See the list of GEs in the FI-WARE catalogue in [5]. In addition, the FI-WARE architecture supports the co-existence of various Web service technologies, as well as the resource-oriented, request/reply and event driven architectural styles.

The ENVIROFI conceptual architecture was built by profiling the various GEs according to the needs of the environmental domain, and also by identifying and specifying the functions specific to EIS applications. Furthermore, as the environmental domain heavily relies on generic geospatial standards, it was essential in the ENVIROFI conceptual architecture design (see Fig. 5) to accommodate these standards on top of the GEs, or better, integrate them among the GEs cluster. 


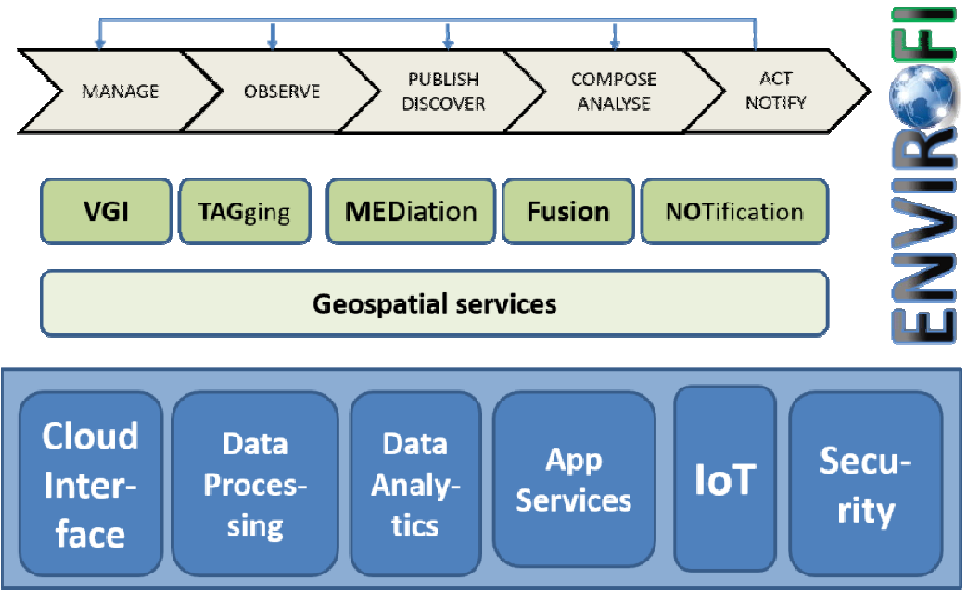

\author{
Environmental \\ Monitoring and \\ Decislon Support \\ Lifecycle \\ Environmental \\ Specific Enablers \\ Geospatial \\ Specific Enablers
}

Fig. 5. ENVIROFI Conceptual Architecture

The specification of the ENVIROFI Conceptual Architecture [4] results from the work and experience of European research projects such as ORCHESTRA ${ }^{4}, \mathrm{SANY}^{5}$, EO2HEAVEN $^{6}$, SWING $^{7}$, ENVISION $^{8}$, EuroGEOSS ${ }^{9}$ and TRIDEC $^{10}$, and relies upon the following pillars:

- Reference models originally specified by standardization organizations such as ISO, OGC, OMG, OASIS and The Open Group, and tailored to the environmental domain [1].

- Profiles of geospatial services and information models specified by ISO and OGC.

- Specifications and implementations of architectural styles, design patterns and (partial) solutions.

A generic Environmental Monitoring and Decision Lifecycle based upon the INSPIRE lifecycle perspective served as a framework for the generalization of use case functionality from the various environmental pilot applications. ENVIROFI contributed with extensions to standardization under CEN /TC289 TR 15449 on Spatial Data Infrastructure, Reference model [6] and Service Centric view [7], and to ISO/TC211 19119 Geospatial Services [8] and further input to the related OGC Topic 12 on Service Architecture. ENVIROFI has also contributed to examples of the use of an RM-ODP modeling approach by use case-based specification templates.

\footnotetext{
${ }^{4}$ Open Architecture and Spatial Data Infrastructure for Risk Management.

${ }^{5}$ Sensors Anywhere.

${ }^{6}$ Earth Observation and Environmental Modelling for the Mitigation of Health Risks.

${ }^{7}$ Semantic Web Services Interoperability for Geospatial decision making.

${ }^{8}$ ENVIronmental Services Infrastructure with ONtologies.

${ }^{9}$ European approach to GEOSS (Global Earth Observation System of Systems).

${ }^{10}$ Collaborative, Complex and Critical Decision-Support in Evolving Crisis.
} 


\section{$5 \quad$ Geospatial and Environmental Enablers}

In order to cope with the domain-independent geospatial aspects, ENVIROFI explicitly identified a set of specific enablers (SE) that address geospatial data provisioning and storage. These geospatial enablers encompass known geospatial information models (e.g. the OGC Observation and Measurements Model) and services (e.g. the OGC Sensor Observation Service). Hence, they may also be reused in other usage areas that deal with geo-referenced data processing, e.g. risk and crisis management [12].

The environmental SEs have been identified under five major categories which are derived from very distinct enablement functionalities ${ }^{11}$ :

1. Harvesters, Connectors and Mediators: These constitute a collection of brokers, connectors and mediator services which support protocols and data models. The models are specialized in the environmental domain. This thematic class of specific enabler facilitates easier interoperability between specific enabler based services, using agile and flexible service implementations.

2. Volunteered Geographic Information: This specializes in geo-referenced observation and data sampling the environmental usage area. The services in this thematic class provide ways to record and archive geo-tagged measurements for later use by other classed of specific enablers. The enablers in this class are designed to support crowdsourcing and crowdtasking of environmental measurements, performed by a large-scale community of volunteering environmental observers.

3. Semantic Tagging Tools: These tools and services provide support for semantic enrichment of environmental data streams and sources. This thematic class includes environmental domain ontology support, harvester services and linked data services, which allow uncertainty annotation of existing measurement resources.

4. Fusion Tools for Heterogeneous Data Sources: These are composed by environmental data fusion services, which may operate at different semantic levels. This thematic class includes pre-processing, feature extraction, situation assessment and forecasting services, with those for preparing and aggregating environmental data into formats which are suitable for use by end users and FI downstream services.

5. Event detection and notification services: These include services, which provide a variety of notification mechanisms, which are compatible with the environmental geospatial standards and protocols.

Fig. 6 below illustrates an example of a KML file which is produced as output using a chain of environmental enablers, which led to the fusion of environmental observations (in this example sea surface temperature) using three semantically conflicting and asynchronous heterogeneous data sources such as in situ, space-borne and model data [10].

${ }^{11}$ See the list of SEs in the ENVIROFI catalogue [9]. 


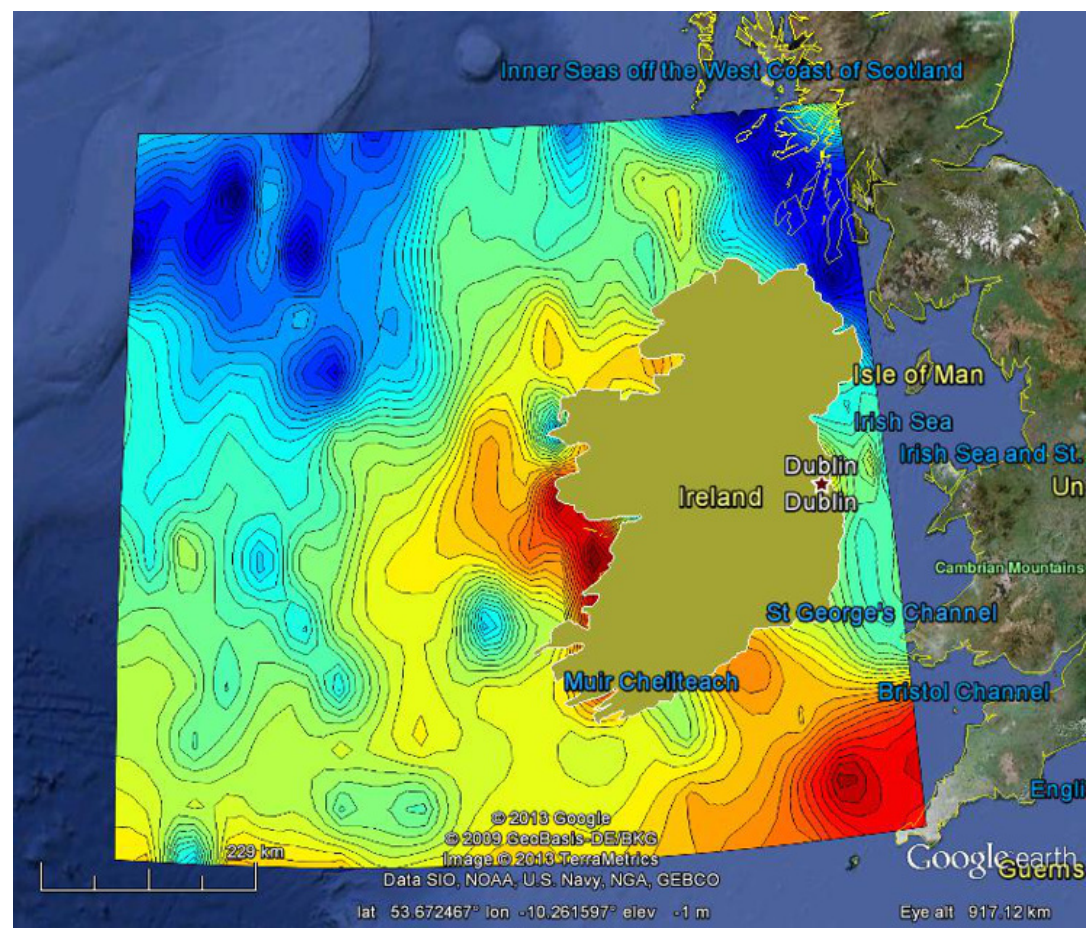

Fig. 6. Future Internet enablement of water quality monitoring in the marine environment, using generic data fusion from multiple observation sources

The above mentioned categories of specific enablers for the environmental domain are also designed as application agnostic as much as possible. They offer the possibility to extend their usage as specific enablers for the environmental domain in its broadest sense and beyond.

\section{Generic Enablers and Cross-Domain Geospatial Environments}

Many of the existing and upcoming GEs from the FI-WARE Cloud Hosting, Data/Context management, and Security chapters are suitable for "filling the (standardization) gaps" in the environmental usage area. Others, most notably the GEs of the IoT chapter to some extent overlap with some types of OGC services.

The main challenge in adopting the GEs for real-world environmental domain applications is the harmonization and integration across the GE chapters. A potential customer needs one contact institution and one technically harmonized platform to take sustainable design decisions. Unfortunately, the initial release of the FI-WARE platform featured GEs at very different levels of maturity, and as a whole lacked harmonization and integration across the GE chapters. For example, the GEs did not support the authentication and authorization functionality of the identity management GE. This is expected to 
change with the second release of the software and the introduction the FI-WARE Open Innovation Laboratory (OIL) later in the year 2013.

Another important challenge of the FI-WARE platform is to address the weak support for geospatial data and processing. This is most notable at the level of event processing, likely to slow down the uptake of the FI-WARE event processing framework in the environmental usage area, and cannot be fully solved without extending the GE functionality. The underlying OMA-NGSI ${ }^{12}$ data model is generic enough to allow the embedding of geospatial information, but the FI-WARE architecture does not define the way to do this. Likewise, the architecture does not prevent geospatial processing, but no standardized geospatial processing functions are defined for existing FI-WARE GEs from the Data/Context management Chapter.

It is also important to understand that FI-WARE neither defines a standardized way for encapsulation of the processing services, nor a consistent set of standard data retrieval services. To a certain extent, this deficiency is compensated by the Publish/Subscribe Context broker GE and the Mediator GE [5]:

- The Mediator GE is defined as a middleware application responsible for providing interoperability among different communication protocols and among different data models. However, the ability to mediate between standardized OGC services used in the environmental usage area and the services used in other domains were not tested in ENVIROFI.

- The Pub/Sub Context Broker GE allows "Content providers" to register and publish information in form of the OMA-NGSI compliant events ("Context Entities"). "Context consumers" can subscribe to registered data sources and consume this information. The Pub/Sub context broker GE is essentially an event broker, but the Context Entities remain cached on the server until pre-defined "expiration time" and can be accessed over RESTful service interface. We have successfully used this property of Pub/Sub context broker to exchange data between the Environmental Georeferenced Observation service SE [9] and the Wirecloud Application Mashup GE in an extension of the biodiversity pilot.

In order to cope with the domain-independent geospatial aspects, ENVIROFI explicitly identified a set of enablers that address geospatial data provisioning and storage. These geospatial enablers encompass the geospatial information models (e.g. the OGC Observation and Measurements Model) and services (e.g. the OGC Sensor Observation Service). Hence, they may also be reused in other usage areas that require geo-referenced data and data processing, e.g. security, transport and logistics, health, risk and crisis management and so forth. With the exception of the applicationspecific models, most of the ENVIROFI enablers provide application and even usage area neutral functionality which involves observing, publishing, accessing and analyzing geospatially and temporally referenced events and measurements. Fig. 7 below illustrates the potential usage of ENVIROFI SEs in various cross-domain usage areas

12 Open Mobile Alliance Next Generation Services Interface, see http://technical.openmobilealliance.org/Technical/ release_program/ngsi_v1_0.aspx 
of the evolving Future Internet. Indeed, further use cases and scenarios may be described to benefit from the FI-WARE and ENVIROFI architecture, and clearly put in value the enablers and technologies being developed over the project [11].

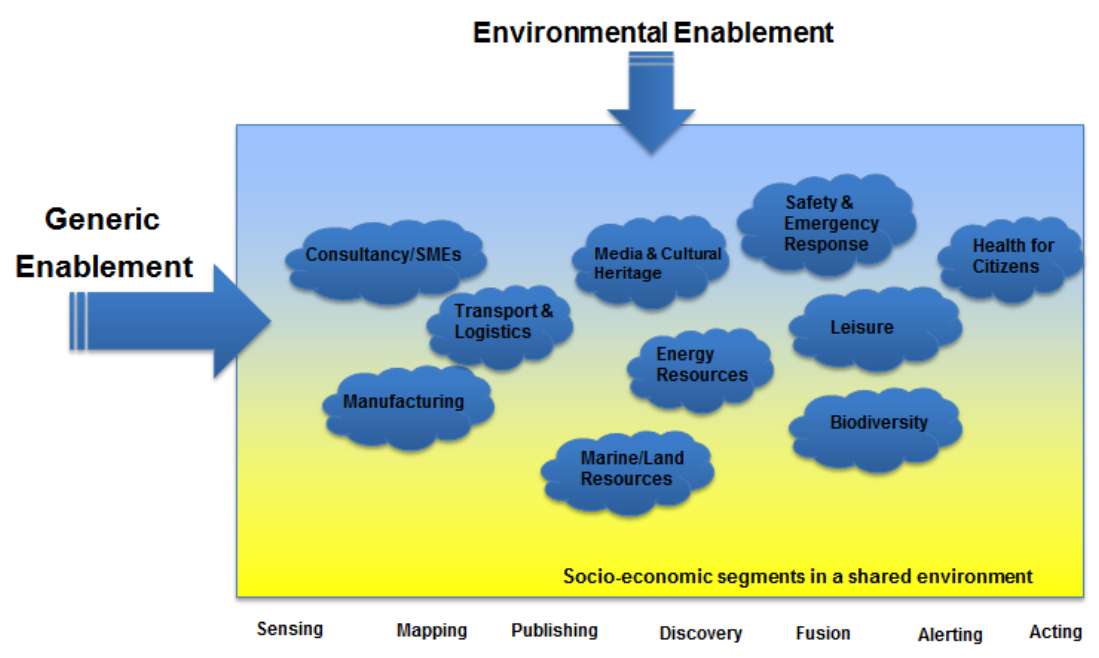

Fig. 7. Vision of the Environmental Enablement in Future Internet Cross-domain Areas

\section{Conclusion}

Despite the current weak support for geospatial data and processing, the ENVIROFI project team considers most of the FI-WARE GEs as generic enough and welcomes the extensions of currently available software building blocks. Many of the existing and upcoming GEs are suitable for "filling the (standardization) gaps" in the environmental usage area. The combination of GEs and environmental SEs could thus be used to build or support many cross-domain scenarios in the FI-PPP large-scale trials in phase 3 and beyond.

Together with SEs from other usage areas, the FI-WARE platform has the potential of becoming one of the important commodities and technology accelerators for European Small to Medium Enterprise organizations. In this sense it will be a major contributor in the growth and expansion of ICT-driven enterprises in the ever growing global digital market. However, the real value and success of FI-WARE generic enablement will greatly depend on its ability to deliver working, intuitive and easy-touse enablers. These should not only connect to the specific enablers of the usage areas but also take on board the geospatial and intelligent data processing requirements in FI experimentations under the various phases of the FI-PPP and beyond. Only then, the creation of sustainable ICT driven ecosystems with robust business and value chains will be achieved. 


\section{References}

1. Usländer, T.: Service-oriented Design of Environmental Information Systems. PhD thesis of the Karlsruhe Institute of Technology (KIT). KIT Scientific Publishing (2010), http: / / digbib.ubka. uni-karlsruhe.de/voll texte/1000016721

2. Schade, S., Fogarty, B., Kobernus, M., Schleidt, K., Gaughan, P., Mazzetti, P., Berre, A.: Environmental Information Systems on the Internet: A Need for Change. In: Hřebíček, J., Schimak, G., Denzer, R. (eds.) ISESS 2011. IFIP AICT, vol. 359, pp. 144-153. Springer, Heidelberg (2011)

3. Usländer, T., Batz, T.: How to Analyse User Requirements for Service-Oriented Environmental Information Systems. In: Hřebíček, J., Schimak, G., Denzer, R. (eds.) ISESS 2011. IFIP AICT, vol. 359, pp. 161-168. Springer, Heidelberg (2011)

4. ENVIROFI Consortium (Ed.). D4.2 Environmental Architecture. Deliverable D4.2 of the FP7 project ENVIROFI Work Package 4 (2012),

http: / / www . envirofi . eu/Downloads / PublicDeliverables / tabid/ 4983 / Default.aspx

5. FI-WARE Catalogue. All information, documentation and tools you need as a developer to start using a Generic Enabler (GE) Implementation, http: //catalogue.fi-ware.eu/

6. CEN/TC289 - TR 15449 Geographic information - Spatial Data Infrastructures - part 1 Reference model (November 2011)

7. CEN/TC289 - TR 15449 Geographic information - Spatial Data Infrastructures - part 4, Service Centric view (May 2012)

8. ISO/TC211 - ISO 19119 PT/EC revision draft (May 2013)

9. ENVIROFI Catalogue. Central repository for implementations of Specific Enablers (SE) for the environmental Usage Area within the Future Internet Public Private Partnership programme (FI-PPP), http: / / catalogue.envirofi.eu /

10. Sabeur, Z., Chakravarthy, A., Bashevoy, M., Modafferi, S.: Structured Multi-level Data Fusion and Modelling of Heterogeneous Environmental Data for Future Internet Applications. Geophysical Research Abstracts, Vol. 15, EGU2013-6311-2. EGU General Assembly (2013)

11. ENVIROFI Consortium (Ed.). D6.2.3 Report on Community Consultation. Deliverable D6.2.3 of the FP7 project ENVIROFI Work Package 6 (2013), http: / /bscw.fi-ppp.eu/pub/bscw.cgi/d17436/ENVIROFI\%20D6.2.3_ Report_on_Community_Consultation_III.pdf

12. Berre, A.J., Usländer, T., Schade, S.: Identification and Specification of Generic and Specific Enablers of the Future Internet - Illustrated by the Geospatial and Environmental Domain. In: Abramowicz, W., Llorente, I.M., Surridge, M., Zisman, A., Vayssière, J. (eds.) ServiceWave 2011. LNCS, vol. 6994, pp. 278-289. Springer, Heidelberg (2011) 